\title{
Treatment of Methamphetamine withdrawal in trauma patients
}

\author{
Phillips B*, Snyder S, Safa H, Wu J, Shahid M, Turco L, Schroeder A, Weber W, O'Connor JA and Asensio JA \\ Department of Surgery, Department of Clinical and Translational Science, Creighton University School of Medicine, Creighton University Medical Center, Omaha,
} Nebraska, USA

\begin{abstract}
Background: Methamphetamine use has increased in the United States over the past several decades. Similarly, an increasing number of trauma patients are testing positive for methamphetamine, which impacts hospital resources and length of stay, particularly in intensive care units. Treatment of methamphetamine-positive patients has not been well defined in the medical literature.

Methods: A systematic literature search was conducted to identify a treatment algorithm and medications used in methamphetamine-intoxication and withdrawal. PRISMA guidelines were followed in the search and classification of the available literature. Inclusion criteria were defined as "any peer-reviewed study that reported results regarding the management and treatment of withdrawal from methamphetamine. All studies that were published in English were initially identified and screened for inclusion. However, due to the paucity of published reports on this subject, all sources were reviewed.

Results: The literature search yielded 329 publications, of which 22 were included in this systematic literature review. A total of 12 randomized trials, 6 animal models and 1 consensus recommendation by the WHO met inclusion criteria. 3 other studies were used as additional reference and supportive evidence. A proposed treatment algorithm for managing methamphetamine withdrawal was not identified. A consensus regarding treatment of methamphetamine-positive patients does not currently exist in the literature. Few drugs have shown any clinical efficacy in managing methamphetamine-withdrawal. However, some drugs indicated potential benefits in animal models. Most prominently, $n$-acetylcysteine, bupropion, and dextroamphetamine, might be beneficial for use in the acute and long-term management of methamphetamine withdrawal.
\end{abstract}

Limitations: The greatest limitation was the lack of available Level I, II, and III studies in the medical literature. Reports that are published include mostly subjective experiences.

Conclusions: The current methamphetamine epidemic impacts medical care and resources at trauma centers in the United States. A consensus regarding treatment of methamphetamine-positive patients, including medicinal agents and timing of their administration is lacking. Additional studies are needed to identify an effective treatment algorithm.

\section{Background}

According to the Substance Abuse and Mental Health Services Administration (SAMHSA), there has been a significant increase of methamphetamine use nationwide [1]. Methamphetamine is a psychostimulant drug that can drastically impair judgment, leading to violent conduct, risk-taking behavior, and an increased rate of intentional or unintentional injuries. Trauma patients testing positive for methamphetamine are primarily involved in motor vehicle accidents, blunt assaults, and gunshot wounds [2]. Those suffering an injury while under the effects of methamphetamine are more likely to be admitted to an intensive care unit (ICU) and are subject to a greater number of diagnostic procedures compared to methamphetaminenegative patients [3,4]. Swanson documented higher rates of laparotomy in methamphetamine-positive patients due to challenges in obtaining a reliable physical examination [3].

From 2003 to 2005, admission rates of methamphetamine-positive patients to trauma centers increased by nearly $70 \%$. Interestingly, methamphetamine-positive trauma patients tend to have lower overall injury severity scores (ISS), but longer hospital stay and higher overall cost of care than methamphetamine-negative patients [2,5]. Methamphetamine use in trauma patients results in greater resource utilization that is out of proportion to injury severity [5].
Current clinical recommendations for withdrawal management from stimulants such as methamphetamine are limited. Recommendations from the World Health Organization (WHO) include symptomatic relief with intravenous fluids, diazepam for agitation, and transfer to psychiatric facilities for extreme agitation [6]. Transfer of trauma patients to inpatient psychiatry for complications related to methamphetamine use is not always feasible. The care of patients undergoing withdrawal from methamphetamine often requires use of restraints and potent chemical sedation. A universally accepted treatment algorithm for methamphetamineintoxication and dependence is lacking. The demand for treatment of methamphetamine-dependence had an eight-fold increase from 1992 to 2005 [7]. However, in the past decade, this demand has

Correspondence to: Bradley J Phillips, Vice Chairman of Surgery - Surgical Research, Associate Professor of Surgery, Department of Surgery, Department of Clinical and Translational Science, Creighton University School of Medicine Creighton University Medical Center, 601 North 30th Street, Suite 3701, Omaha, NE 68131-2137, USA, Tel: 402-717-4909; Fax: 402-717-6068; E-mail: Bradley. Phillips@alegent.org

Key words: methamphetamine, withdrawal, treatment, nutrition

Received: February 07, 2017; Accepted: February 22, 2017; Published: February 25,2017 
specifically increased in trauma centers. Our working group attempted to establish a literature consensus and identify treatment protocols and recommendations specifically for methamphetamine-positive trauma patients.

\section{Methods}

A PRISMA-based approach was utilized in this search of the literature. The paucity of published reports led to consideration of all available sources based on relevancy. The search was conducted through PubMed, using the key phrases "methamphetamine \& treatment", "methamphetamine \& mental illness", "methamphetamine \& amantadine", "methamphetamine toxicity", "methamphetamine withdrawal", "methamphetamine alcohol withdrawal protocol", "methamphetamine withdrawal treatment," "methamphetamine and supplements," and "methamphetamine and nutrition." All studies written in English were selected based on a review of the title and abstract. Studies that focused on management of methamphetaminepositive trauma patients testing were filtered. Regardless of publication date, all studies were considered. Studies were further categorized into case reports, animal models, clinical trials, and literature reviews.

\section{Results}

Our literature search yielded 276 studies for the words "methamphetamine withdrawal treatment", of which 22 were selected based on our selection approach. A treatment algorithm for medical management of methamphetamine intoxication and withdrawal was not identified. Indications for treating withdrawal symptoms with medicinal agents or hospital admission, such as threshold vital signs or specific symptomologies, were not identified. A consensus on treatment of methamphetamine-positive patients does not currently exist in the literature and few drugs have shown clinical efficacy. Most agents that have been studied are not superior to placebo in managing methamphetamine withdrawal. Drugs that have shown some clinical efficacy have not been corroborated in large follow-up studies. The available animal models reported in the literature show potential, however these have not advanced to clinical trial applications.

\section{Discussion}

This systematic literature review aimed at identifying a treatment consensus and medications used in methamphetamine-intoxication and withdrawal, specifically in trauma patients. A widely accepted consensus regarding treatment of methamphetamine-positive patients and withdrawal does not currently exist in the literature. Few drugs have shown any clinical efficacy in managing methamphetaminewithdrawal and there is only a limited number of randomized trials available on this topic [8]. However, we identified clinical trials showing promising results for the acute medical management of methamphetamine-positive patients.

$\mathrm{N}$-acetylcysteine (NAC), a drug used to treat acetaminophen overdose and IV contrast toxicity, has also been found to decrease methamphetamine cravings. In one controlled, double-blinded randomized study, patients receiving $1200 \mathrm{mg}$ per day of oral NAC over a four-week period had decreased cravings for methamphetamine compared to placebo $(\mathrm{P}<0.001)$ [9]. This drug may prove useful in the acute setting.

Bupropion, a dopamine reuptake inhibitor used to treat depression and nicotine dependence, is thought to increase synaptic concentrations of dopamine. Chronic methamphetamine use results in low dopaminergic tone [10]. One randomized, placebo-controlled trial of bupropion in methamphetamine dependence found that $150 \mathrm{mg}$ BID of oral bupropion increased abstinence from methamphetamine in low to moderate users [11]. Bupropion is fairly well-tolerated with limited side effects of insomnia, dry mouth, and nausea. In a study of 151 patients, bupropion has also been shown to produce nearlystatistically significant differences in methamphetamine abstinence when compared to a placebo $(p=0.09)$ [12], possibly leading to decreased recidivism in an outpatient setting.

Modafinil is a glutamate enhancer which produces many contradictory effects of stimulant withdrawal, such as increased energy, attention, wakefulness, and locomotion, while improving mood and reducing appetite [13]. Patients dependent on cocaine who were treated with modafinil reported less use $(\mathrm{p}=0.03)$ when compared to a placebo group [13]. Applying this to the use of methamphetamine, the drug may assist with the dysphoria characteristic of methamphetamine withdrawal. Modafinil may also decrease craving for methamphetamine while improving overall cognition [13] and is currently being investigated in a number of clinical trials.

Dextroamphetamine has also shown some benefit in decreasing methamphetamine cravings and withdrawal symptoms [14]. Researchers have used $60 \mathrm{mg}$ of oral sustained-release dextroamphetamine over a period of 8 weeks to achieve these results. Dextroamphetamine may play a role in the recovery and rehabilitation of an injured patient through a mechanism similar to bupropion.

Gamma-vinyl GABA (GVG), an antiepileptic that prevents breakdown of GABA, has shown efficacy in sustaining abstinence from methamphetamine [15]. In a study evaluating abstinence, 16 of 18 participants completing the 9-week study tested negative for methamphetamine and cocaine. GVG was started at $500 \mathrm{mg}$ twice daily for days 1-3 and increased to $1.5 \mathrm{~g}$ once daily for days $4-7$ and $2 \mathrm{~g}$ once daily for days $8-14$. On day 15 , subjects were placed on $3 \mathrm{~g}$ once daily for 4 weeks, and then tapered to zero over the next 3 weeks, for a total of 137 grams over the entire study. Although GVG is associated with changes in visual acuity, it was not observed in the study [16].

Antipsychotics have also been proposed to aid in withdrawal management. In one study, risperidone was administered at $3.6 \mathrm{mg} /$ day and resulted in decreased methamphetamine use and significantly improved fine motor functioning after 30 days $(p=0.001)$ [17]. Antipsychotic medications can manage symptoms such as irritability, agitation, depression, increased sleeping, increased appetite, and muscle aches. Antipsychotics have been showed to resolve these symptoms within one week after discontinuation of stimulants such as methamphetamine [6]. This lends support for antipsychotics having potential use in trauma patients with prolonged hospital stays.

Rivastigmine is an acetylcholinesterase inhibitor studied for use in methamphetamine-positive patients. Acetylcholine has been linked to the reinforcing and psychomotor activating effects of methamphetamine [10] thus, an acetylcholinesterase inhibitor may decrease such behaviors. Rivastigmine has reduced methamphetamineassociated increases in diastolic blood pressure and self-reported feelings of anxiety as well as the desire for more drugs [10].

Other agents have limited efficacy in managing methamphetaminepositive patients. Mirtazapine has been shown in one study to be well tolerated, although participants reported myalgia more frequently than those treated with modafinil. Of note, modafinil was noted to cause less sleep disturbance than mirtazapine [18]. Another study of mirtazapine revealed that it may lessen anxiety and hyperarousal caused by methamphetamine withdrawal; however, other investigations have 
failed to duplicate these results [19]. Imipramine has not shown any significant benefits in managing cocaine/methamphetamine-abusing patients [20].

Ondansetron, a 5- $\mathrm{HT}_{3}$ receptor antagonist and modulator of corticomesolimbic dopamine, functions through its low affinity for dopamine receptors [21] and has shown potential benefit in outpatient settings. Some data in an animal-based investigation suggested that the combination of ondansetron with pergolide, ergoline-based dopamine receptor, may serve as a useful treatment for methamphetamine abuse by reducing relapse rates [22]. Sertraline, a selective serotonin reuptake inhibitor has proven to be useful to an extent. When combined with contingency management, sertraline allowed more patients to abstain from methamphetamine when compared to patients treated with sertraline alone. Contingency management involves rewarding of good behavior and implementation of operant conditioning and reinforcement, which can be used to support drug abusers [23]. However, in patients with symptoms of depression, sertraline did not have an improved outcome compared to placebo, and actually resulted in increased methamphetamine use and more frequent relapse $(\mathrm{p}<0.05)[23]$.

Lobeline may be very useful for the treatment of stimulant abusers, including those of methamphetamine. It may interact and stimulate the vesicular monoamine transporter (VMAT) [24], as well as the cell surface dopamine transporter (DAT), which are both action sites for methamphetamine [24]. Lobeline has long been used to aid in smoking cessation and has a low abuse potential [25]. A study showed that lobeline induced dopamine release in mammalian cells expressing dopamine transport proteins DAT and VMAT-2. In methamphetamine users, dopamine release may reduce overall cravings [26].

Memantine, a non-competitive antagonist of the N-methyl-Daspartate receptor (NMDA glutamate receptor), was used in a 2010 study with methamphetamine-intoxicated rats.

This study found that memantine inhibited methamphetaminetriggered behavior and reversed overall anxiety [27]. Adverse effects of methamphetamine can be potentially treated with memantine in an attempt to prevent cognitive deficits.

Animal studies provide a plethora of information regarding certain drug treatments for methamphetamine withdrawal. For example, one study tested the usefulness of propofol in methamphetamineintoxicated rats and found reduced cerebral oxidative stress and swelling and improved mitochondrial function [28]. Another study showed the protective role of melatonin on the blood brain barrier in methamphetamine-induced inflammatory responses [29]. More clinical trials will be necessary to investigate the potential of these drugs for the treatment of methamphetamine-withdrawal.

\section{Limitations}

This systematic literature search was limited by the paucity of Level I, II, and III studies regarding treatment of methamphetamineintoxication and withdrawal.

\section{Conclusion}

This systematic review of the literature did not reveal a consensus on the management of methamphetamine-positive patients in the hospital setting. However, several agents have shown potential clinical benefit and can be considered for use in the treatment of this challenging patient population. Management in the acute setting should target cravings, decompensation, lability, and aggression. Based on current literature, $\mathrm{N}$-acetylcysteine at $1200 \mathrm{mg} /$ day appears to be a reasonable option. Oral sustained-release dextroamphetamine at $60 \mathrm{mg}$ could be used for symptoms of withdrawal. Long-term treatment with sustained-release bupropion $150 \mathrm{mg}$ twice daily could be initiated after hospital discharge if there is adequate follow-up to monitor its effects. Further investigation is required before any recommendations can be made regarding potential use of other drugs such as modafinil, risperidone, rivastigmine, and GVG. Overall, substance abuse is a rapidly expanding problem affecting our population. More well designed trials are needed to formulate a clear consensus regarding appropriate management and treatment of methamphetamine-positive patients requiring hospital admission.

\section{References}

1. Substance Abuse and Mental Health Services Administration. NSDUH Estimated Totals by State. SAMSA. 2013-2104.

2. London JA, Utter GH, Battistella F, Wisner D (2009) Methamphetamine use is associated with increased hospital resource consumption among minimally injured trauma patients. J Trauma 66:485-490. [Crossref]

3. Hadjizacharia P, Green DJ, Plurad D, Chan LS, Inaba K, et al. (2009) Methamphetamines in Trauma: Effect on Injury Patterns and Outcome. J Trauma66:895-898.[Crossref]

4. Swanson SM, Sise CB, Sise MJ, Sack DI, Holbrook TL, et al (2007) The scourge of methampheramine: impact on a level I trauma center. J Trauma63: 531-537.[Crossref]

5. Tominaga GT, Garcia G, Dzierba A, Wong J (2004) Toll of methamphetamine on the trauma system. Arch Surg139:844-847.[Crossref]

6. WHO (2009) Clinical Guidelines for Withdrawal Management and Treatment of Drug Dependence in Closed Setting.

7. KM Kampman (2008) The Search for Medications to Treat Stimulant Dependence. Addict SciClinPract4: 28-35.[Crossref]

8. Hoffmann L, Schumann N, Fankhaenel T, Thiel C, Klement A, et al. (2016) Methamphetamine use in Central Germany: protocol for a qualitative study exploring requirements and challenges in healthcare from the professionals' perspective. $B M J$ Open6:e11445.

9. Mousavi SG, Sharbafchi MR, Salehi M, Peykanpour M, KarimianSichani N, et al. (2015) The efficacy of N-acetylcysteine in the treatment of methamphetamine dependence: a double-blind controlled, crossover study. Arch Iran Med 18: 28-33. [Crossref]

10. Montoya ID, Vocci F (2008) Novel Medications to Treat Addictive Disorders. Curr Psychiatry Rep 10: 392-398.[Crossref]

11. Shoptaw S1, Heinzerling KG, Rotheram-Fuller E, Steward T, Wang J, E et al. (2008) Randomized, placebo-controlled trial of bupropion for the treatment of methamphetamine dependence. Drug Alcohol Depend 96:222-32. [Crossref]

12. Elkashef AM, Rawson RA, Anderson AL, Li SH, Holmes T, et al. (2008) Bupropion for the treatment of methamphetamine dependence. Neuropsychopharmacology 33:1162-1170.[Crossref]

13. Dackis CA, Kampman KM, Lynch KG, Pettinati HM, O'Brien CP (2005) A double-blind, placebo-controlled trial of modafinil for cocaine dependence. Neuropsychopharmacology 30:205-211.[Crossref]

14. Galloway G, Buscemi R, Coyle J, Flower K, Siegrist J, et al. (2011) Randomized, Placebo-Controlled Trial of Sustained-Release Dextroamphetamine for Treatment of Methamphetamine Addiction. ClinPharmacolTher89: 276-282. [Crossref]

15. Brodie J, Figueroa E, Dewey S (2003) Treating Cocaine Addiction: From Preclinical to Clinical Trial Experience with gamma-vinyl GABA. Synapse 50: 261-265.[Crossref]

16. Brodie J, Figueroa E, Laska E, Dewey S (2004) Safety and Efficacy of gamma-vinyl GABA (GVG) for the Treatment of Methamphetamine and/or Cocaine Addiction. Synapse55: 122-125.[Crossref]

17. Meredith CW, Jaffe C, Yanasak E, Cherrier M, Saxon AJ (2007) An Open-label Pilot Study of Risperidone in the Treatment of Methamphetamine Dependence. $J$ Psychoactive Drugs 39: 167-172.[Crossref]

18. Mcgregor C, Srisurapanont M, Mitchell A, Wickes W, White, J (2008) Symptom and sleep patterns during inpatient treatment of methamphetamine withdrawal: A comparison of mirtazapine and modafinil with treatment as usual. $J$ Subst Abuse Treat35: 334-342.[Crossref] 
19. Shoptaw SJ, Kao U, Heinzerling K, Ling W (2009) Treatment for amphetamine withdrawal. Cochrane Database Syst Rev 15:CD003021.[Crossref]

20. Galloway G, Newmeyer J, Knapp T, Stalcup S, Smith, D (1994) Imipramine for the treatment of cocaine and methamphetamine dependence. J Addict Dis 13:201-216. [Crossref]

21. Johnson B, Ait-Daoud N, Elkashef A, Smith E, Kahn R, et al. (2008) A preliminary randomized, double-blind, placebo-controlled study of the safety and efficacy of ondansetron in the treatment of methamphetamine dependence. Int $J$ Neuropsychopharmacol11: 1-14. [Crossref]

22. Davidson C, Gopalan R, Ahn C, Chen Q, Mannelli P, et al. (2007) Reduction in methamphetamine induced sensitization and reinstatement after combined pergolide plus ondansetron treatment during withdrawal. Eur J Pharmaco565:113-118.[Crossref]

23. Shoptaw S, Huber A, Peck J, Yang X, Liu J, et al. (2006) Randomized, placebocontrolled trial of sertraline and contingency management for the treatment of methamphetamine dependence. Drug Alcohol Depend 85:12-18.[Crossref]

24. Teng L, Crooks PA, Sonsalla PK, Dwoskin LP (1997) Lobeline and nicotine evoke $[3 \mathrm{H}]$ overflow from rat striatal slices preloaded with $[3 \mathrm{H}]$ dopamine: differential inhibition of synaptosomal and vesicular $[3 \mathrm{H}]$ dopamine uptake. $J$ PharmacolExpTher280:1432-1444.[Crossref]
25. Dwoskin LP, Crooks PA (2002) A novel mechanism of action and potential use for lobeline as a treatment for psychostimulant abuse. BiochemPharmacol63:89-98. [Crossref]

26. Wilhelm CJ, Johnson RA, Eshleman AJ, Janowsky A (2008)Lobeline effects on tonic and methamphetamine-induced dopamine release. BiochemPharmacol75: 1411-1415. [Crossref]

27. Camarasa J, Rodrigo T, Pubill D, Elena Escubedo E (2010)Memantine Is a Useful Drug to Prevent the Spatial and Non-spatial Memory Deficits Induced by Methamphetamine in Rats. Pharmacol Res 62: 450-56. [Crossref]

28. Shokrzadeh M, Zamani E, Mehrzad M, Norian Y, Shaki F (2015) Protective Effects of Propofol Against Methamphetamine-induced Neurotoxicity. ToxicolInt22: 92-99. [Crossref]

29. Jumnongprakhon P, Govitrapong P, Tocharus C, Tocharus J (2016) Melatonin promotes blood-brain barrier integrity in methamphetamine -induced inflammation in primary rat brain microvascular endothelial cells. Brain Research646:182-192.

Copyright: $\subset 2017$ Phillips B. This is an open-access article distributed under the terms of the Creative Commons Attribution License, which permits unrestricted use, distribution, and reproduction in any medium, provided the original author and source are credited. 